\title{
Driven Tunneling: New Possibilities for Coherent and Incoherent Quantum Transport
}

\author{
T. Dittrich, P. Hänggi, B. Oelschlägel, and R. Utermann \\ Institut für Physik, Universität Augsburg, Memminger Straße 6, \\ D-86135 Augsburg, Germany
}

\begin{abstract}
We study the conservative as well as the dissipative quantal dynamics in a harmonically driven, quartic double-well potential. In the deep quantal regime, we find coherent modifications of tunneling, including its complete suppression. In the semiclassical regime of the conservative system, the dynamics is dominated by the interplay of tunneling and chaotic diffusion. A strong correlation exists between the tunnel splittings and the overlaps of the associated doublet states with the chaotic layer. With weak dissipation, remnants of coherent behaviour occur as transients, such as the tunneling between symmetry-related pairs of limit cycles. The coherent suppression of tunneling observed in the conservative case is stabilized by weak incoherence. The quantal stationary states are broadened anisotropically due to quantum noise, as compared to the corresponding classical attractors.
\end{abstract}

\section{Introduction}

During the last decades one observes a strong tendency, both in experimental and theoretical physics, to shift focus from a global, macroscopic point of view towards the microscopic study of moderately small systems - nanometerscale electronic devices, molecules, small metallic clusters. For such a system in a nonequilibrium environment, there are typically three components which together make up the essential physics: A coherent driving which represents the macroscopic energy source and can often be described classically, the microscopic system itself, with its dynamics characterized by the simultaneous presence of quantum effects and classical nonlinearity, and an environment comprising a large number of weakly coupled degrees of freedom which serve as a sink both for energy and coherent information.

In the present contribution we intend to give an overview over the interplay of these three components, for a specific example: a bistable system driven by a harmonic force. Bistability is an elementary source of nonlinear behaviour [1-5]; 
with a periodic driving, it enables an enormously rich dynamical repertoire [6-9]. At the same time, bistable systems provide a paradigm for quantum coherence: tunneling [10]. A harmonic driving captures the essence of a ubiquitous energy source, electromagnetic irradiation, specifically of lasers and their relatives.

We shall approach the complexity of weakly dissipative, semiclassical nonlinear behaviour in three steps: Following an introduction into the model in Sect. 2, its dynamics in the deep quantal regime is outlined in Sect. 3. Section 4 is devoted to the semiclassical regime of the conservative dynamics. In Sect. 5, weak dissipation is brought into play. Section 6 contains a summary and an outlook.

The present work forms a synopsis of results partially published elsewhere [11-18].

\section{The model and its symmetries}

We formulate the harmonically driven bistable system as a quartic double well with a spatially homogeneous, classical sinusoidal driving force. It is described by the Hamiltonian

$$
H_{\mathrm{DW}}(x, p ; t)=H_{0}+H_{1}, \quad H_{0}(x, p)=\frac{p^{2}}{2}-\frac{1}{4} x^{2}+\frac{1}{64 D} x^{4}, \quad H_{1}(x ; t)=x S \cos \omega t .
$$

With the dimensionless variables used, the only parameter controlling the unperturbed Hamiltonian $H_{0}(x, p)$ is the barrier height $D$. It approximately gives the number of doublets with energies below the top of the barrier. Accordingly, the classical limit amounts to $D \rightarrow \infty$.

The symmetry of the Hamiltonian under discrete time translations, $t \rightarrow$ $t+2 \pi / \omega$, enables to use the Floquet formalism [19-22], which generalizes most of the conceptual tools of spectral analysis to the present context. Its basic ingredient is the Floquet operator, i.e., the unitary propagator that generates the time evolution over one period of the driving force, $U=\operatorname{Texp}\left(-\mathrm{i} \int_{0}^{2 \pi / \omega} \mathrm{d} t H_{\mathrm{DW}}(t) / \hbar\right)$, where $T$ effects time ordering. Its eigenvectors and eigenphases are referred to as Floquet states and quasienergies, respectively. Being phases, the quasienergies are organized in classes, $\epsilon_{\alpha, k}=\epsilon_{\alpha}+k \omega, k=0, \pm 1, \pm 2, \ldots$, where each member corresponds to a physically equivalent solution. Therefore, all spectral information is contained in a single "Brillouin zone", $-\omega / 2 \leq \epsilon<\omega / 2$.

Besides invariance under time translation and time reversal, the unperturbed system possesses the spatial reflection symmetry $x \rightarrow-x, p \rightarrow p, t \rightarrow t$. For the specific time dependence of a harmonic driving as it is used here, the symmetry $f(t+\pi / \omega)=-f(t)$ restores a similar situation as in the unperturbed case: The system is now invariant against the operation $[11,23] \mathrm{P}: p \rightarrow-p, x \rightarrow$ $-x, t \rightarrow t+\frac{\pi}{\omega}$, which may be regarded as a generalized parity in the extended, three-dimensional phase space spanned by $x, p$, and time $t \bmod (2 \pi / \omega)$. As in the unperturbed case, this enables to separate the eigenstates into an even and an odd subset. 


\section{Driven tunneling and localization}

To give an impression of driven tunneling in the deep quantal regime, we study how a state, prepared as a coherent state centered in the left well, evolves in time under the external force. Since this state is approximately given by a superposition of the two lowest unperturbed eigenstates, $|\Phi(0)\rangle \approx\left(\left|\Psi_{1}\right\rangle+\left|\Psi_{2}\right\rangle\right) / \sqrt{2}$, its time evolution is dominated by the Floquet-state doublet originating from $\left|\Psi_{1}\right\rangle$ and $\left|\Psi_{2}\right\rangle$, and the splitting $\epsilon_{2}-\epsilon_{1}$ of its quasienergies.

There are two regimes in the $(\omega, S)$-plane where tunneling is not qualitatively altered by the external force: Both in the limits of slow (adiabatic) and of fast driving, the separation of the time scales of the inherent dynamics and of the external force effectively uncouples the two processes and results in a mere renormalization of the tunnel splitting $\Delta$. Both an analytical treatment and numerical experiments show that the driving always reduces the effective barrier height and thus increases the tunneling rate in these two limits [11].

Qualitative changes in the tunneling behavior are expected as soon as the driving frequency becomes comparable to the internal frequencies of the double well, in particular, to the tunnel splitting and to the so-called resonances $E_{3}-E_{2}$, $E_{4}-E_{1}, E_{5}-E_{2}, \ldots$ By spectral decomposition, the temporal complexity in this regime is immediately related to the "landscape" of quasienergy planes $\epsilon_{\alpha, k}(\omega, S)$ in parameter space. Features of particular significance are close encounters of quasienergies: Two quasienergies cross one another without disturbance if they belong to different parity classes, otherwise they form an avoided crossing.

The transition at $\omega=E_{3}-E_{2}$, a single-photon transition in the terminology of quantum optics, is called fundamental resonance. For $S>0$, the corresponding quasienergies $\epsilon_{2, k}$ and $\epsilon_{3, k-1}$ form an avoided crossing, since they have equal parity. Fig. la shows the time evolution of the probability to return, $P^{\Phi}\left(t_{n}\right)=\left|\left\langle\Phi(0) \mid \Phi\left(t_{n}\right)\right\rangle\right|^{2}$ at the fundamental resonance. The monochromatic oscillation of $P^{\Phi}\left(t_{n}\right)$ characteristic of unperturbed tunneling has given way to a more complex beat pattern. Its Fourier transform reveals that it is composed mainly of two groups of three frequencies each (Fig. 1b), which can be associated with the allowed transitions among the Floquet states pertaining to the two lowest doublets.

In contrast, a two-photon transition that bridges the tunnel splitting $\Delta$ is "parity forbidden", and thus the quasienergies $\epsilon_{2, k-1}$ and $\epsilon_{1, k+1}$ can form exact crossings. A vanishing of the difference $\epsilon_{2,-1}-\epsilon_{1,1}$ will have a remarkable consequence: For a state prepared as a superposition of the corresponding two Floquet eigenstates only, $P^{\Phi}(t)$ and all other observables become constants, at least at the discrete times $t_{n}$, and thus it is possible that tunneling comes to a standstill. According to an argument going back to von Neumann and Wigner [24], exact crossings should occur along one-dimensional manifolds in the $(\omega, S)$ plane. In the present case, there is one such manifold $S_{\mathrm{loc}, k}(\omega)$ for each condition $\epsilon_{2,-k}=\epsilon_{1, k}$. Fig. 2 a shows $S_{\text {loc }, 0}(\omega)$ : a closed curve, reflection-symmetric with respect to the line $S=0$. A typical time evolution of $P^{\Phi}\left(t_{n}\right)$ for a parameter point on the linear part of that manifold is presented in Fig. 2 b. It clearly 

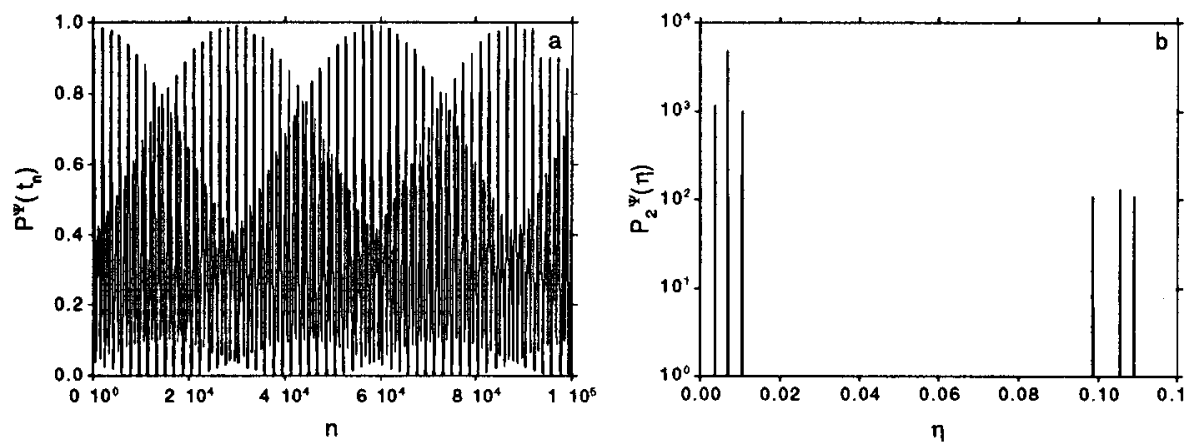

Fig. 1. Driven tunneling at the fundamental resonance $\omega=E_{3}-E_{2}$. (a) Time evolution of $P^{\Phi}\left(t_{n}\right)$ over the first $2 \times 10^{5}$ time steps; (b) local spectral two-point correlation function $P_{2}^{\Phi}(\eta)$ obtained from (a). The parameter values are $D=2, S=2 \times 10^{-3}$, and $\omega=0.876$.
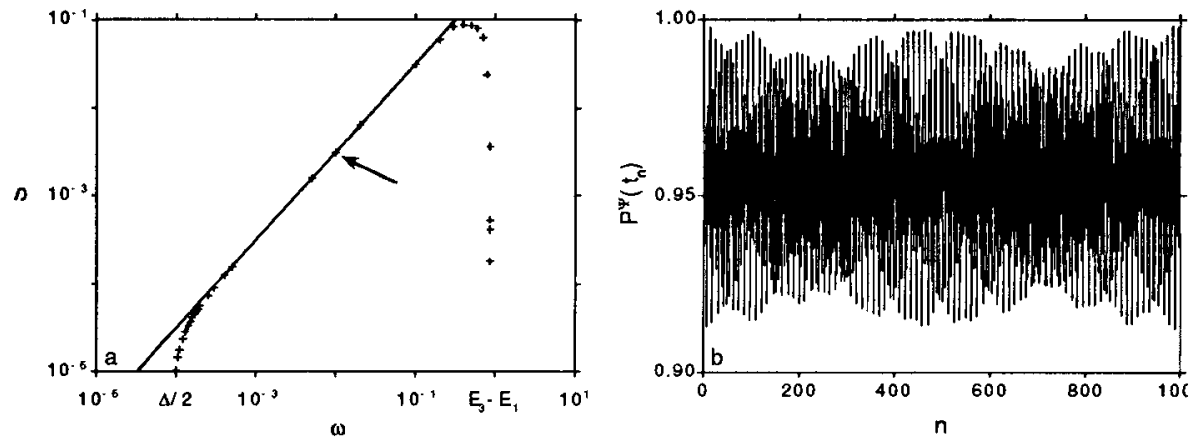

Fig. 2. Suppression of tunneling at an exact crossing $\epsilon_{2,-1}=\epsilon_{1,1}$. (a) The manifold $S_{\text {loc, }, 0}(\omega)$ in the $(\omega, S)$ plane where this crossing occurs (data obtained by diagonalization of the full Floquet operator are indicated by crosses, the full line is based on a two-state approximation $[13,25,26]$, the arrow indicates the parameter point to which part (b) refers); (b) time evolution of $P^{\Phi}\left(t_{n}\right)$ over the first $10^{3}$ time steps, at $D=2$, $S=3.171 \times 10^{-3}, \omega=0.01$.

demonstrates that tunneling is almost completely suppressed. The remaining oscillations of small amplitude can be ascribed to an admixture of higher-lying quasienergy states to the initial state. The suppression of tunneling is an elementary quantum-interference effect, much of which can be understood on basis of a two-state approximation $[13,25,26]$. 


\section{Tunnel splittings and the onset of chaos}

In the classical double well, the most significant consequence of the periodic driving is the onset of deterministic chaos [27]. It develops around the hyperbolic fixed point at the top of the barrier and along the separatrix originating there. For sufficiently high $S$, deterministic diffusion along the separatrix becomes a significant contribution to the classical phase-space transport. Quantum mechanically, it competes with tunneling [28-33]. Applying ideas from EinsteinBrillouin-Keller (EBK) quantization for periodically driven systems [34] and from random-matrix theory for mixed (regular and chaotic) systems [35] to the present context, one obtains the following crude picture of the impact of the chaotic layer on tunneling [29-31]: Even with the driving, the two isolated regular regions within the wells remain related by the generalized parity $P$. Accordingly, Floquet states residing within these regions form a ladder of tunnelsplitted doublets. For states mainly residing within the chaotic layer, in contrast, random-matrix theory predicts level repulsion. We therefore expect that, as soon as one of the pairs of quantizing tori pertaining to the symmetry-related regular regions resolves in the spreading chaotic layer, the exponentially small splitting of the corresponding doublet breaks up and eventually reaches a size of the order of the mean level separation. As a consequence, the coherent tunneling on an extremely long time scale will give way to a more irregular dynamics on shorter time scales, forming the quantal counterpart of deterministic diffusion along the separatrix. The breakup of the tunnel doublets in the chaotic layer is not a direct consequence of the Lyapunov exponent of the classical dynamics being positive there, but rests on the global condition that diffusive spreading connects all parts of the chaotic layer, bridging the symmetry plane.
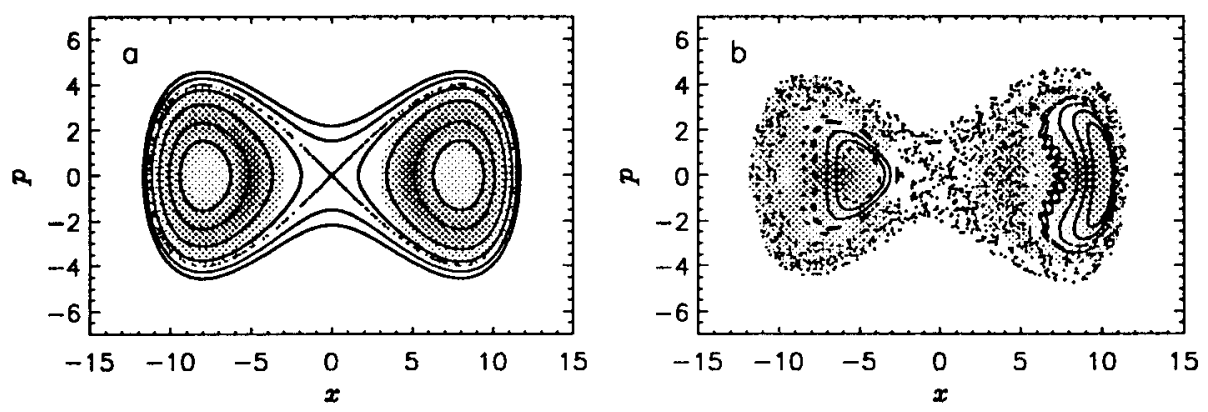

Fig. 3. Husimi distributions (in gray-scale representation) for the quasienergy state $\left|\phi_{7}(0)\right\rangle$, compared with the corresponding classical phase-space portraits, at (a) $S=$ $10^{-5}$ and (b) $S=0.2$.

In order to allow for a numerical check, we quantify the distinction between "regular" and "chaotic" eigenstates on the basis of the overlap of the Husimi 

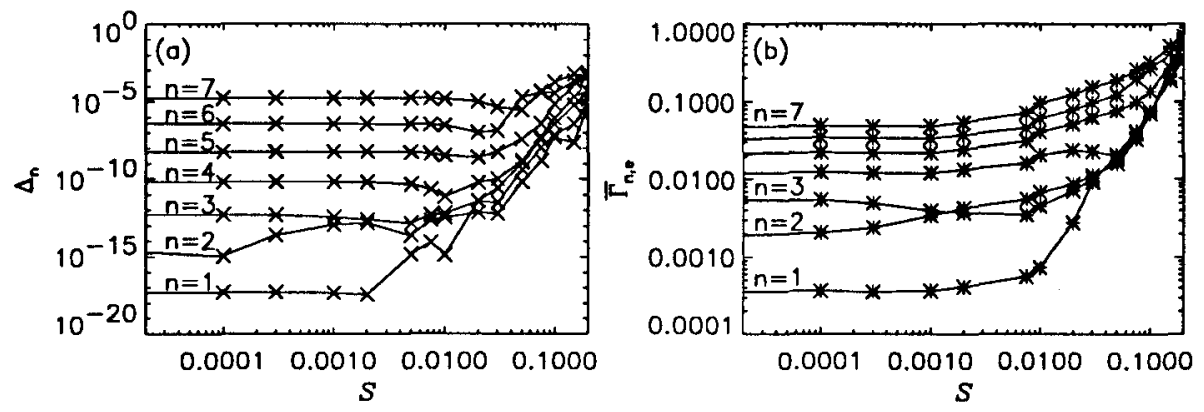

Fig. 4. Tunnel splittings (a) and overlaps with the chaotic layer (b) for the seven lowest tunnel doublets, as functions of the amplitude of the driving.

representation [36] of an eigenstate with the chaotic layer in phase space $[17,18]$. To illustrate these concepts, the Husimi distribution for the quasienergy state $\left|\phi_{7}\right\rangle$ is superposed, in Fig. 3, with the corresponding classical phase space portrait, for (a) $S=10^{-5}$ and for (b) $S=0.2$, at phase 0 of the driving. In Fig. 4, we compare the $S$ dependencies of the tunnel splittings and the overlaps for the seven quasienergy doublets from $\left|\phi_{1}\right\rangle,\left|\phi_{2}\right\rangle$ to $\left|\phi_{13}\right\rangle,\left|\phi_{14}\right\rangle[17,18]$. Qualitatively, they are strikingly similar: There is only a weak $S$ dependence, reflecting the influence of the growing first resonance, for $S \leq 10^{-3}$. For $S \gtrsim 10^{-3}$, both the tunnel splittings and the overlaps start to grow exponentially, one by one, starting from the lowest doublet, so that the range of these quantities reduces by several orders of magnitude. The $S$ regime of this steep increase coincides with that of the onset of chaotic motion in the classical dynamics. Insofar, the picture sketched above is confirmed. Details, however, need to be revised.

The notion that each splitting widens individually as the corresponding quantizing torus resolves, is not unambiguously corroborated by the data. It would imply that the transitions to a large splitting occur "from top to bottom", i.e., first for the doublet localized on the outermost torus pair within the separatrix. If this transition is assessed from the splittings passing a certain absolute threshold, e.g., $\Delta_{\lambda}=10^{-4}$, that order is roughly obeyed. If, however, the point of onset of exponential growth, visible in a logarithmic plot like Fig. 4a, is taken as the criterion, the order is reversed. Another noticeable fact is that the widening of the splittings and the concomitant change in character of the eigenstates, as functions of $S$, are continuous processes that can only roughly be associated with the decay of a KAM torus, taken as a discrete event. Even doublet states overlapping by $70 \%$ with the chaotic layer may still show a relatively small splitting and exhibit the signature of a regular state in their spatial structure and time dependence. It is not clear whether this retarded decay of the tunnel doublets corresponds to the gradual disintegration of classical tori via cantori [37] and vague tori $[38]$. 


\section{Driven tunneling with dissipation}

In this section, we extend our working model (1), such as to include the influence of dissipation and noise on the microscopic level, following the usual procedure of coupling the central system to a heat bath $[39,40]$. Proceeding in a similar way as in Ref. [41], we use the density operator in the Floquet basis, reduced to the double-well degree of freedom, as the basis of our description, and resort to the usual rotating-wave and Markov approximations. This allows us to derive the equation of motion for the density matrix $\tilde{\sigma}$ (in the interaction picture with respect to $H_{\mathrm{I}}$ ) in the form of the master equation [41]

$$
\begin{aligned}
\dot{\sigma}_{\alpha, \beta}(t)= & \delta_{\alpha, \beta} \sum_{\nu}\left(W_{\alpha, \nu} \tilde{\sigma}_{\nu, \nu}(t)-W_{\nu, \alpha} \tilde{\sigma}_{\alpha, \alpha}(t)\right)+ \\
& \frac{1}{2}\left(1-\delta_{\alpha, \beta}\right) \sum_{\nu}\left(W_{\nu, \alpha}+W_{\nu, \beta}\right) \tilde{\sigma}_{\alpha, \beta}(t) .
\end{aligned}
$$

It comprises a closed subset of equations for the evolution of the diagonal elements towards a steady state, and another subset describing the decay of the non-diagonal elements. The coefficients $W_{\alpha, \beta}$ depend on the coupling constants and on the quasienergies; they are given elsewhere [16]. The classical limit of the dissipative quantal dynamics (specifying a linear frequency dependence of the coupling strength with cutoff) leads to a Langevin equation describing a bistable Duffing oscillator [6-9] showing Ohmic damping, with a macroscopic damping constant $\gamma$, and fluctuations.

A particularly interesting question is whether the coherent suppression of tunneling observed in the conservative case will survive in the presence of dissipation. In order to obtain an adequate description of this phenomenon on basis of a master equation like (2), the rotating-wave approximation must be avoided. It is valid only if the time scales of the classical relaxation and of the conservative quantal dynamics are clearly separated. However, in the vicinity of the manifolds $S_{\text {loc,k }}(\omega)$ where the tunnel splitting vanishes (see Fig. 2a), exceedingly small energy scales and correspondingly large time scales occur in the undamped dynamics. This necessitates to take also quasienergy transitions into account that virtually violate energy conservation. Details of this refinement of the master equation are given in Refs. [16,42].

Fig. 5a shows the time evolution of $P^{\sigma}\left(t_{n}\right)=\operatorname{tr}\left[\sigma\left(t_{n}\right) \sigma(0)\right]$ (the analogue of $P^{\Phi}\left(t_{n}\right)$ for a density-matrix representation) at a parameter point very close to, but not exactly on $S_{\text {loc, } 0}(\omega)$, for $\gamma=10^{-6}$ and various values of $T$. For low temperature, $P^{\sigma}\left(t_{n}\right)$ exhibits a slowly decaying coherent oscillation with a very long period, due to the slight offset from $S_{\text {loc, }}(\omega)$ [16]. Asymptotically, the distribution among the wells is completely thermalized. With increasing temperature, the decay time of the slow coherent oscillation first decreases until this oscillation is suppressed from the beginning (the corresponding part of the graph is not shown in Fig. 5b). After going through a minimum, however, the thermalization time increases again. At a characteristic temperature $T^{*}$, this time scale reaches a resonance-like maximum where the incoherent processes 

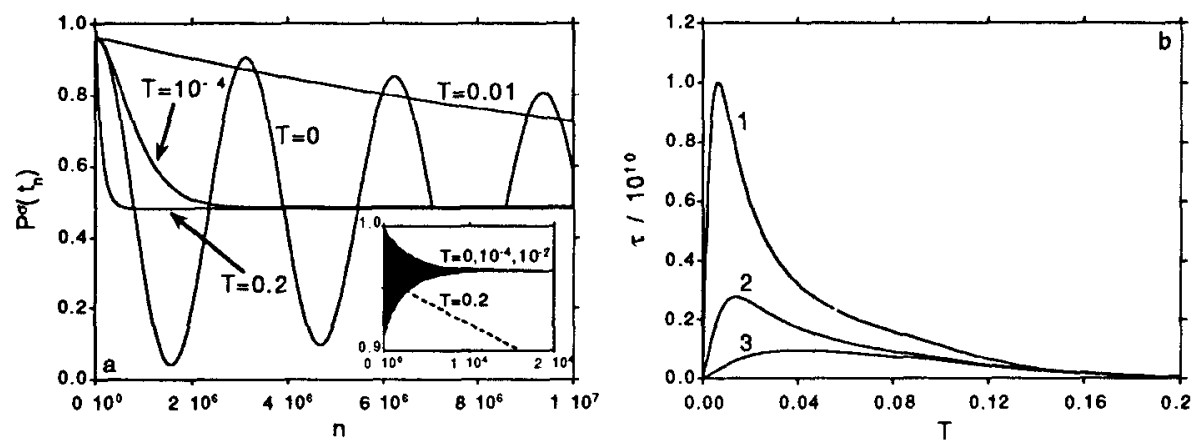

Fig. 5. Coherent suppression of tunneling in the presence of weak dissipation. (a) Time evolution of $P^{\sigma}\left(t_{n}\right)$ over the first $10^{7}$ time steps, at a parameter point $(D=2, \omega=0.01$, and $S=3.171 \times 10^{-3}$ ) close to $S_{\mathrm{loc}, 0}(\omega)$ (see Fig. $2 \mathrm{a}$ ), for $\gamma=10^{-6}$ and various values of $T$, starting from a pure, minimum-uncertainty state centered in one of the wells; (b) temperature dependence of the decay time $\tau$ of $P^{\sigma}\left(t_{n}\right)$ for three values of the detuning $\Delta \omega$ from the manifold $S_{\text {loc }, 0}(\omega)$ (graph 1: $\Delta \omega=-1.4 \times 10^{-7}$, as in part (a), 2: $\Delta \omega=5.0 \times 10^{-7}$ at $S=3.1712 \times 10^{-3}, 3: \Delta \omega=1.4 \times 10^{-6}$ at $S=3.1715 \times 10^{-3}$ ). The other parameters are as in part (a). The data shown do not extend down to $T=0$, where $\tau(T)$ diverges, but start only with the rising part of this function.

induced by the reservoir stabilize the localization of the wave packet and thus compensate for the detuning introduced deliberately. In Fig. 5b, we present the temperature dependence of the decay time $\tau$ (defined by $P^{\sigma}\left(t_{n}\right) \sim \exp (-n / \tau)$ ) for three values of the detuning $\Delta \omega$ from the manifold $S_{\text {loc, } 0}(\omega)$. A variation of $\gamma$ reveals that the dependence on the damping constant has a similar resonance-like form [42].

This stabilization of the coherent suppression of tunneling by noise is to be distinguished from the trivial localization by strong damping. In fact, it has already been observed in a model analogous to (1), but with the deterministic harmonic driving of the double well replaced by a noisy one, so that the time evolution remained unitary and a damping could not occur [14]. Rather, this phenomenon bears some resemblance to the quantum zeno effect in a bistable system [43], and to the classical stabilization of instable equilibrium states by multiplicative noise $[44,45]$.

Besides discussing the influence of dissipation on coherence effects, one may conversely ask how the classical dynamics of the driven damped Duffing oscillator [6-9] is modified by quantal interference. A hallmark of the classical dynamics is the existence of attractors of various degrees of complexity, as a function of $\omega, S$, or $\gamma$. In Fig. 6 [42], we choose parameter values where classically, there exist five limit cycles, with the frequency of the driving: two symmetry-related pairs with one partner within each well, and a single one encircling the wells. The Husimi 
distribution in the stationary state at phase 0 is overlayed with the phase-space portrait of the corresponding conservative classical system (in a periodically driven system, the stationary state may still possess a time dependence with the period of the driving, which however is invisible in a stroboscopic plot like this). Both the classical attractors and the maxima of the quantal stationary distribution, while not coinciding exactly, are located near elliptic fixed points of the conservative dynamics and can be associated with the regular regions around the potential minima and the first resonance, respectively (the fifth limit cycle outside the wells is not significantly populated in the quantal stationary state). The quantum noise preferentially broadens the stationary distribution along the limit cycle to which the corresponding classical attractor belongs, the direction in which the classical phase-space flow is least contractive [46].

While the smoothing due to quantum noise is the only quantum effect left in the stationary state, a remnant of coherent tunneling survives in the transient behavior [42]. Fig. 7 shows the time evolution of $P^{\sigma}\left(t_{n}\right)$ for the same parameter values as above, with the initial states prepared as coherent states at the location of either one of the maxima of the asymptotic distribution (see Fig. 6) within the left well, corresponding to nonresonant motion (a) and to the first resonance (b), respectively. In both cases, we observe a coherent oscillation decaying as the stationary state is approached. Fig. 8 reveals that these oscillations form a remnant of tunneling between the partners of each of the two symmetry-related pairs of regular regions, i.e., tunneling between limit cycles. Thermalization within each pair, and subsequently among the pairs, is reached only on longer time scales of the order of the classical relaxation time.

\section{Summary}

The present paper is intended to highlight a number of facettes of a generic nonequilibrium system: The nonlinear quantum dynamics in a periodically driven double-well potential, at different stages of the transition from microscopic, coherent to macroscopic, incoherent behavior. (For the effects of periodic driving on the tunneling decay out of a single metastable state, see Ref. [47].) In the deep quantal regime, we find modifications, due to the driving, of the familiar tunneling. They range from a mere acceleration of its rate, in the two extremes of slow and of fast driving, through complex quantum beats near resonances with the unperturbed system frequencies, to an almost complete suppression of tunneling by a coherent mechanism.

Towards the semiclassical limit of the conservative system, we addressed the interplay between coherent transport by tunneling and diffusive transport along the chaotic layer which develops in the vicinity of the separatrix of the undriven system. Eigenstate doublets residing within the symmetry-related pair of regular regions of the classical phase space exhibit exponentially small splittings and thus support tunneling. As the pair of quantizing tori pertaining to such a doublet resolves in the chaotic sea, the splitting widens and tunneling gives way to a more complex dynamics contributing to the quantal counterpart of chatic diffusion. 


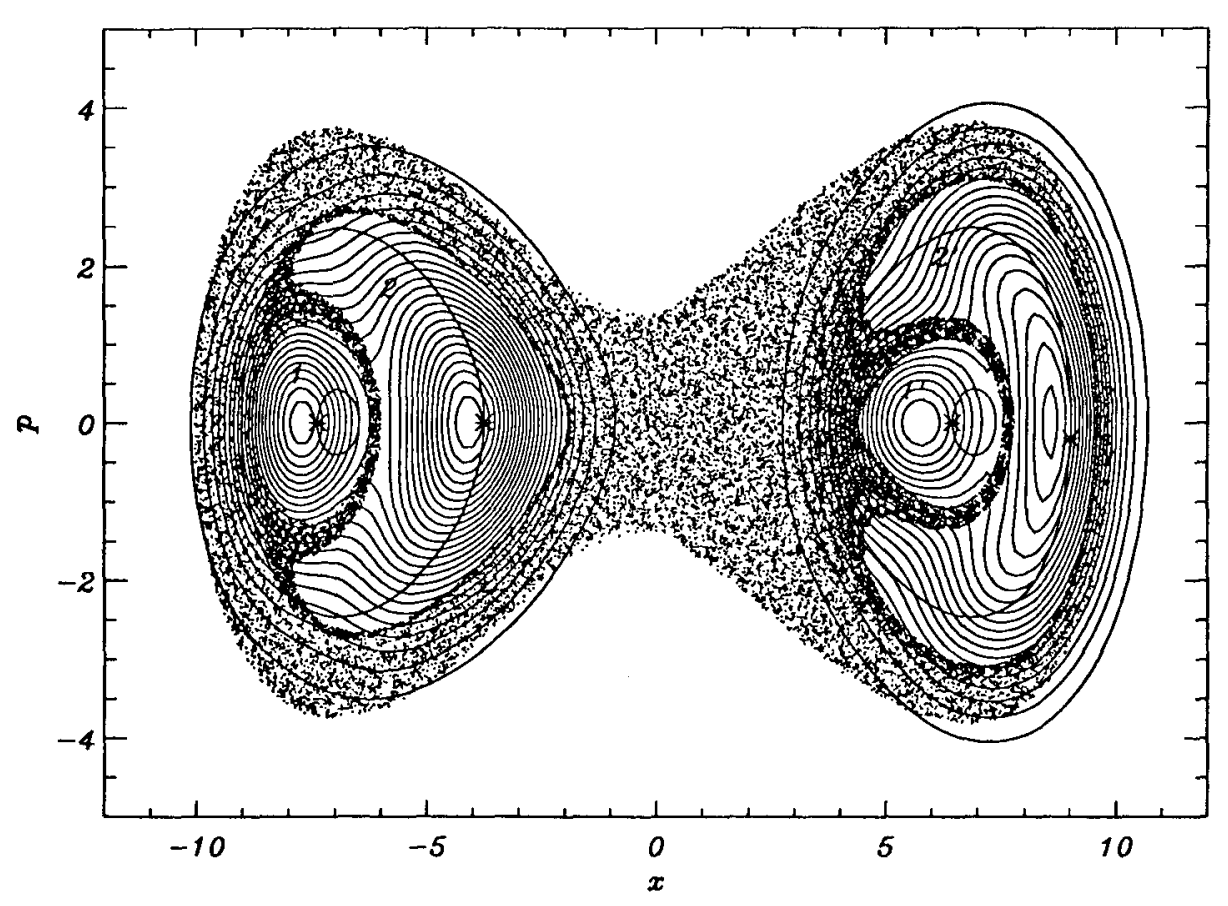

Fig. 6. Quantal stationary state. Contour plot of the asymptotic Husimi distribution at $D=6, S=0.0849, \omega=0.9, \gamma=10^{-5}$, and $T=0$, compared to the limit cycles of the corresponding classical system (the positions on the cycles at phase 0 are indicated by asteriscs), and to the phase-space portrait of the corresponding conservative dynamics.
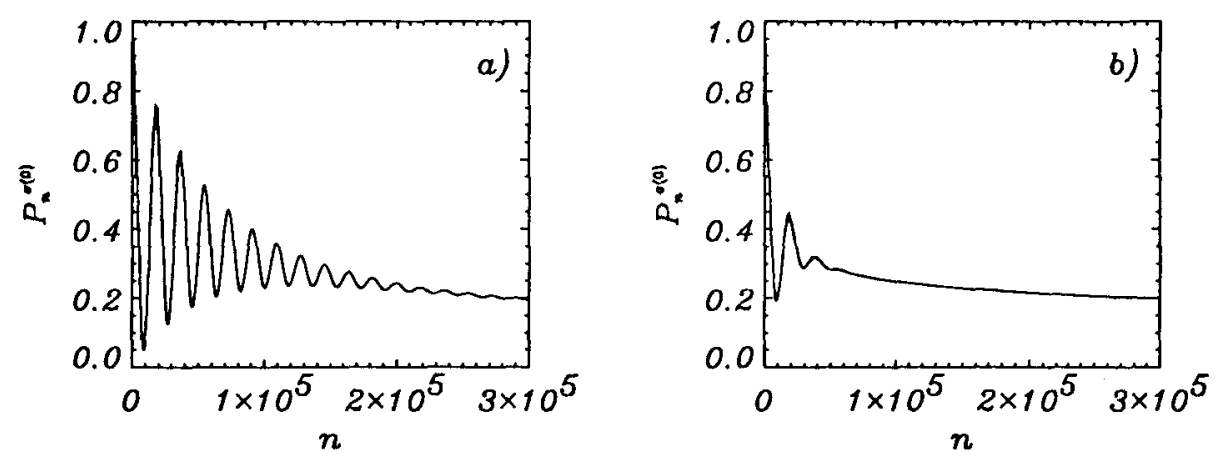

Fig. 7. Transient tunneling between limit cycles, in terms of the time evolution of $P^{\sigma}\left(t_{n}\right)$ over the first $3 \times 10^{5}$ time steps, at paramater values as in Fig. 6 , but with $\gamma=5 \times 10^{-6}$, for initial states prepared as coherent states located at either one of the maxima of the stationary Husimi distribution in the left well, i.e., at $p=0$ and (a) $x=-7.5$, (b) $x=-4.2$. 

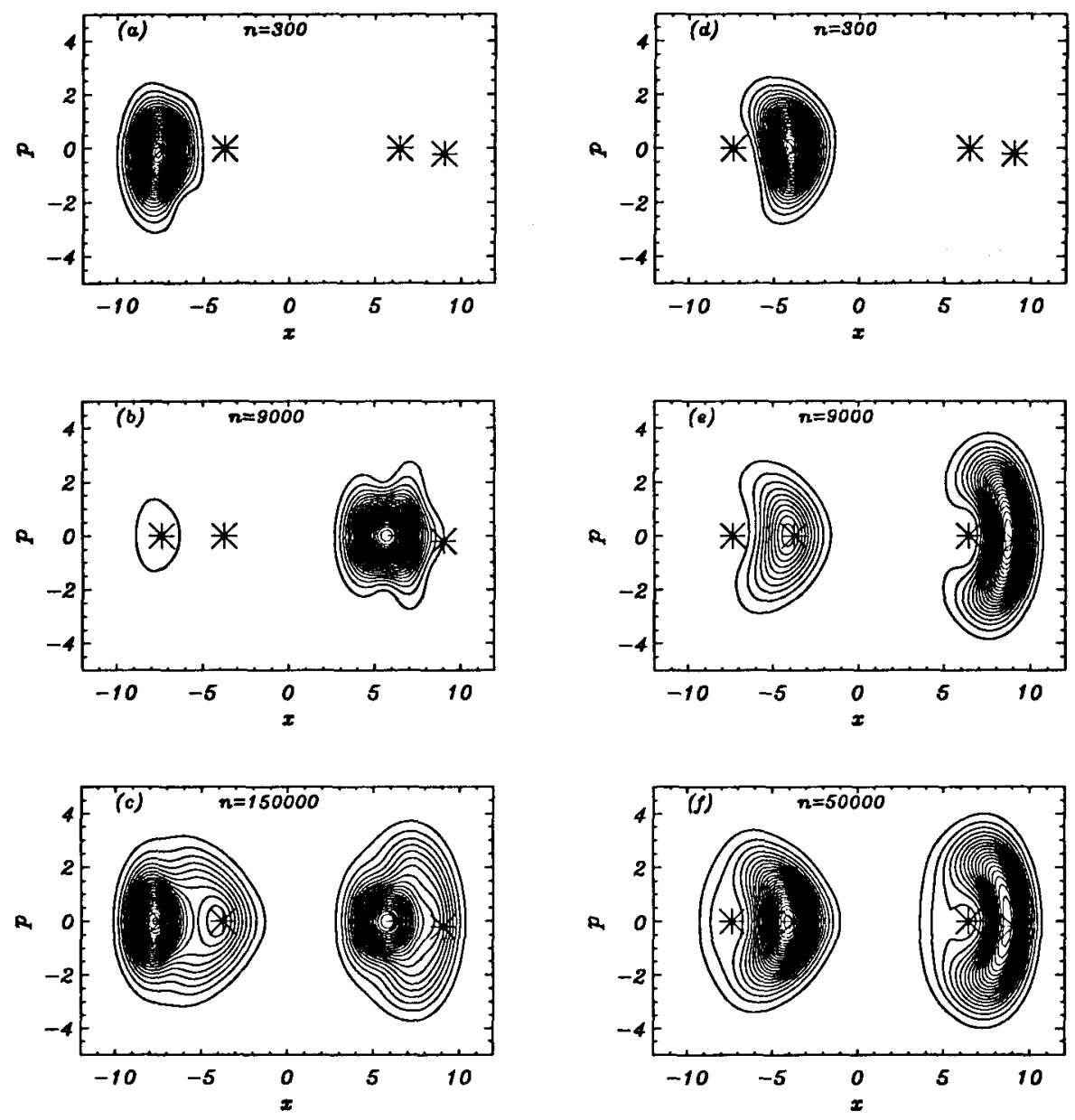

Fig. 8. Transient tunneling between limit cycles, in terms of the Husimi distribution at various times, for parameter values as in Fig. 7 , and initial state for panels $(a-c)$ as in Fig. 7a, for panels $(\mathrm{d}-\mathrm{f})$ as in Fig. $7 \mathrm{~b}$. The positions, at phase 0 , on the limit cycles of the corresponding classical dynamics are indicated by asteriscs.

The disturbing effects of the ambient degrees of freedom render the coherence effects observed in the deep quantal regime transients. Surprisingly, the coherent suppression of tunneling is stabilized if damping constant and temperature take specific values, a result akin to the quantum zeno effect and to the classical stabilization of instable equilibria by multiplicative noise. On the time scale of classical relaxation, the dissipative quantum dynamics approaches a stationary state which forms the analogue of the attractors of the corresponding classical 
dynamics. These stationary states are broadened by quantum noise which is not isotropic, but acts preferentially in the direction where the classical phasespace flow is least contractive, e.g., stronger along limit cycles than transverse to them. On time scales shorter than the classical relaxation, coherence effects such as the tunneling between the basins of attraction of symmetry-related pairs of attractors remain visible.

Many questions have been left open. In the conservative case, an analytical description, in terms of semiclassical concepts, of tunneling in the presence of chaos is not yet available. In the parameter regime of the dissipative system where several strange attractors coexist, both transient tunneling between their basins of attraction, and the quantal smoothing of the fractal basin boundaries [48] should be studied. Finally, it is possible that the phenomenon of stochastic resonance, generated by external classical noise in periodically driven bistable systems [49], can be induced by the inherent quantum noise addressed in Sect. 5 as well.

\section{References}

1 S. Chakravarty and S. Kivelson, Phys. Rev. B 3276 (1985).

2 M. H. Devoret, D. Estéve, J.M. Martinis, A. Cleland, and J. Clarke, Phys. Rev. B 3658 (1987); J. Clarke, A. N. Cleland, M. H. Devoret, D. Estéve, and J. M. Martinis, Science 239992 (1988).

$3 \quad$ R. Bavli and H. Metiu, Phys. Rev. Lett. 691986 (1992).

4 J.E. Combariza, B. Just, J. Manz, and G. K. Paramonov, J. Phys. Chem. 95 10351 (1992).

5 L. M. Sander and H. B. Shore, Phys. Rev. B 31472 (1969).

6 P. Holmes, Philos. Trans. R. Soc. London, Ser. A 292419 (1979).

7 B. A. Huberman and J. P. Crutchfield, Phys. Rev. Lett. 431743 (1979).

8 Y. Ueda, J. Stat. Phys. 20181 (1979); Ann. N. Y. Acad. Sci. 357422 (1980).

9 W. Szemplińska-Stupnicka, Nonlinear Dynamics 3225 (1992).

10 F. Hund, Z. Phys. 43, 803 (1927).

11 F. Grossmann, P. Jung, T. Dittrich, and P. Hänggi, Z. Phys. B 84315 (1991); Phys. Rev. Lett. 67516 (1991); .

12 F. Grossmann, T. Dittrich, and P. Hänggi, Physica B 175293 (1991).

13 F. Grossmann and P. Hänggi, Europhys. Lett. 18571 (1992).

14 F. Grossmann, T. Dittrich, P. Jung, and P. Hänggi, J. Stat. Phys. 70229 (1993).

15 T. Dittrich, F. Grossmann, P. Jung, B. Oelschlägel, and P. Hänggi, Physica A 194173 (1993).

16 B. Oelschlägel, T. Dittrich, and P. Hänggi, Act. Phys. Pol. B 24845 (1993); Europhys. Lett. 225 (1993).

17 R. Utermann, T. Dittrich, and P. Hänggi, Phys. Rev. E 49273 (1994); Physica B 194-196 1013 (1994).

18 T. Dittrich, F. Großmann, P. Hänggi, B. Oelschlägel, R. Utermann, in Proc. of the Third Max Born Symposium Stochasticity and Quantum Chaos, ed. by Z. Haba (Kluwer Academic, Dordrecht, in print).

19 J.H. Shirley, Phys. Rev. 138B 979 (1965).

20 H. Sambe, Phys. Rev. A 72203 (1973). 
N. L. Manakov, V.D. Ovsiannikov, and L. P. Rapoport, Phys. Rep. 141319 (1986).

S. Chu, Adv. Chem. Phys. 73739 (1989).

A. Peres, Phys. Rev. Lett. 67158 (1991).

J. von Neumann and E. Wigner, Phys. Z. 30467 (1929).

J. M. Gomez Llorente and J. Plata, Phys. Rev. A 45 R6958 (1992).

L. Wang and J. Shao, Phys. Rev. A 49 R637 (1994).

L. E. Reichl and W. M. Zheng, in Directions in Chaos, vol. 1, ed. by H. B. Lin (World Scientific, Singapore 1987), p. 17.

M. J. Davis and E. J. Heller, J. Chem. Phys. 75246 (1986).

O. Bohigas, S. Tomsovic, and D. Ullmo, Phys. Rev. Lett. 64, 1479 (1990); ibid. 655 (1990).

S. Tomsovic and D. Ullmo, Tunneling in the Presence of Chaos, preprint 1990.
O. Bohigas, S. Tomsovic, and D. Ullmo, Phys. Rep. 22343 (1993).

W. A. Lin and L. E. Ballentine, Phys. Rev. Lett. 65, 2927 (1990); Phys. Rev. A 453637 (1992).

J. Plata and J. M. Gomez Llorente, J. Phys. A 25 L303 (1992).

H. P. Breuer and M. Holthaus, Ann. Phys. (N. Y.) 211249 (1991).

M. V. Berry and M. Robnik, J. Phys. A 172413 (1984).

K. Husimi, Proc. Phys. Math. Soc. Jap. 22264 (1940).

L. E. Reichl, in The Transition to Chaos: In Conservative and Classical Systems: Quantum Manifestations (Springer, New York 1992), Chaps. 3.9, 9.5.1, and refs. therein.

W. P. Reinhardt, J. Phys. Chem. 86, 2158 (1982); R. B. Shirts and W. P. Reinhardt, J. Chem. Phys. 775204 (1982).

H. Haken, vol. XXV/2c of Encyclopedia of Physics, edited by S. Flügge (Springer, Berlin 1970).

40 W. H. Louisell, Quantum Statistical Properties of Radiation (Wiley, London 1973).

R. Blümel, A. Buchleitner, R. Graham, L. Sirko, U. Smilansky, and H. Walther, Phys. Rev. A 444521 (1991).

B. Oelschlägel, Ph.D. thesis, University of Augsburg, unpublished (1993).

M. J. Gagen, H. M. Wiseman, and G. J. Milburn, Phys. Rev. A 48132 (1993).

R. Graham and A. Schenzle, Phys. Rev. A 261676 (1982).

M. Lücke and F. Schank, Phys. Rev. Lett. 541465 (1985).

T. Dittrich and R. Graham, Europhys. Lett. 4263 (1987); Ann. Phys. (N.Y.) 200363 (1990).

47 F. Grossmann and P. Hänggi, Europhys. Lett. 181 (1992); Chem. Phys. 170 295 (1993).

48 C. Grebogi, S. W. McDonald, E. Ott, and J. A. Yorke, Physics Letters 99A 415 (1983); S. W. McDonald, C. Grebogi, E. Ott, and J. A. Yorke, Physica D 17125 (1985). 